\title{
An unanticipated tumor-suppressive role of the SUMO pathway in the intestine unveiled by Ubc9 haploinsufficiency
}

\author{
Ignacio López $\mathbb{B}^{1}$ - Eleftheria Chalatsi $\mathbb{1}^{1,2,5}$ - Saskia I. J. Ellenbroek $\mathbb{1}^{3}$ - Alexandra Andrieux ${ }^{1}$. \\ Pierre-François Roux $\mathbb{D}^{1}$ - Juan P. Cerapio $\mathbb{I}^{1,6} \cdot$ Grégory Jouvion $\mathbb{1}^{4} \cdot$ Jacco van Rheenen $\mathbb{1}^{3} \cdot$ Jacob-S. Seeler $\mathbb{1}^{1}$. \\ Anne Dejean (10) ${ }^{1}$
}

Received: 23 March 2020 / Revised: 20 August 2020 / Accepted: 4 September 2020 / Published online: 18 September 2020

(c) The Author(s) 2020. This article is published with open access

\begin{abstract}
Sumoylation is an essential posttranslational modification in eukaryotes that has emerged as an important pathway in oncogenic processes. Most human cancers display hyperactivated sumoylation and many cancer cells are remarkably sensitive to its inhibition, thus supporting application of chemical sumoylation inhibitors in cancer treatment. Here we show, first, that transformed embryonic fibroblasts derived from mice haploinsufficient for Ubc9, the essential and unique gene encoding the SUMO E2 conjugating enzyme, exhibit enhanced proliferation and transformed phenotypes in vitro and as xenografts ex vivo. To then evaluate the possible impact of loss of one $U b c 9$ allele in vivo, we used a mouse model of intestinal tumorigenesis. We crossed $U b c 9^{+/-}$mice with mice harboring a conditional ablation of Apc either all along the crypt-villus axis or only in $\mathrm{Lgr}^{+}$crypt-based columnar (CBC) cells, the cell compartment that includes the intestinal stem cells proposed as cells-of-origin of intestinal cancer. While $U b c 9^{+/-}$mice display no overt phenotypes and no globally visible hyposumoylation in cells of the small intestine, we found, strikingly, that, upon loss of Apc in both models, $U b c 9^{+/-}$ mice develop more ( $>2$-fold) intestinal adenomas and show significantly shortened survival. This is accompanied by reduced global sumoylation levels in the polyps, indicating that Ubc9 levels become critical upon oncogenic stress. Moreover, we found that, in normal conditions, $U b c 9^{+/-}$mice show a moderate but robust (15\%) increase in the number of $\operatorname{Lgr} 5^{+} \mathrm{CBC}$ cells when compared to their wild-type littermates, and further, that these cells display higher degree of stemness and cancerrelated and inflammatory gene expression signatures that, altogether, may contribute to enhanced intestinal tumorigenesis. The phenotypes of Ubc9 haploinsufficiency discovered here indicate an unanticipated tumor-suppressive role of sumoylation, one that may have important implications for optimal use of sumoylation inhibitors in the clinic.
\end{abstract}

These authors contributed equally: Ignacio López, Eleftheria Chalatsi

Supplementary information The online version of this article (https:// doi.org/10.1038/s41388-020-01457-y) contains supplementary material, which is available to authorized users.

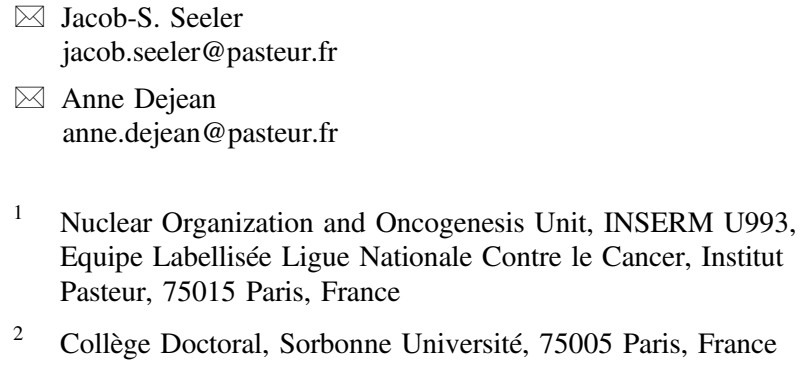

1 Nuclear Organization and Oncogenesis Unit, INSERM U993, Equipe Labellisée Ligue Nationale Contre le Cancer, Institut Pasteur, 75015 Paris, France

2 Collège Doctoral, Sorbonne Université, 75005 Paris, France

\section{Introduction}

SUMO modification has emerged as an essential and highly dynamic posttranslational modification (PTM) that targets hundreds of cellular proteins (the "sumoylome"), thus affecting most, if not all, fundamental processes carried out by eukaryotic cells $[1,2]$. As with other, "small-molecule" PTMs

3 Division of Molecular Pathology, Oncode Institute, The Netherlands Cancer Institute, Amsterdam, The Netherlands

4 Experimental Neuropathology Unit, Institut Pasteur, 75015 Paris, France

5 Present address: Bio-Rad Laboratories, Marnes-la-Coquette, France

6 Present address: Centre de Recherches en Cancérologie de Toulouse, Université de Toulouse, Toulouse, France 
(phosphorylation, acetylation, methylation, etc.), the covalent attachment of the $\sim 11 \mathrm{KDa}$ SUMO peptide changes the interacting properties of its target proteins and hence their functions as parts of larger molecular complexes or pathways.

Numerous stresses and pathological conditions, notably infection and cancer, result in the rapid modification, but also de-modification, of numerous substrates with, at times, profound effects on the composition of the cellular sumoylome [2-7]. Conversely, strong perturbation of the SUMO system by inhibiting the activity of the unique E1 (SAE1/SAE2) or E2 (Ubc9) enzymes, for example, provokes significant cell stress that leads to growth arrest, senescence or apoptosis [8-11].

As with stress, the cancerous state is associated with dysregulated cellular sumoylation dynamics stemming from the altered, mostly upregulated expression of SUMO pathway components (i.e., SUMO, E1, E2, and SENPs) that affects numerous SUMO-targeted cellular factors, including key tumor suppressors and oncoproteins $[6,7]$. The concept of "non-oncogene addiction" $[12,13]$ has been suggested as a possible view of SUMO's roles in cancer: the idea that with strong oncogenic signaling, for example by elevated Myc or Ras activity, the stress-mitigating functions of sumoylation become indispensable for cancer cell survival [14-16]. Clearly, this makes SUMO modification an attractive target for cancer therapy, particularly in cases such as Myc-driven cancers in which the oncoprotein itself remains out of range.

Our understanding of the origins of colorectal cancer (CRC) has greatly benefited from a large body of work, including elegant lineage tracing studies in mice [17]. These studies provided a detailed spatial and temporal description of the rapid, continuous and orderly developmental progression that links the $\mathrm{Lgr}^{+}$intestinal stem cell, Paneth and transit-amplifying cell compartment with the differentiated epithelial cells to constitute the functional crypt-villus anatomy of the small intestinal epithelium. Ninety percent of CRC cases follow a well-defined and ordered chain of genetic events starting with perturbation of APC/ $\beta$-catenin signaling followed by dysregulation of the KRAS, TP53, and/or PIK3CA pathways [18-20]. Significantly, the progression from normal intestinal mucosa, through aberrant crypt foci, small intestinal adenomas or polyps to malignant tumors (but rarely to colonic tumors) is recapitulated in mouse models for the specific and inducible ablation of the Apc tumor suppressor [20]. The characterization of $\mathrm{Lgr}^{+}$ crypt-based columnar (CBC) cells, that include the intestinal stem cells (ISCs) shown to act as cells-of-origin of intestinal cancer [21, 22], attests to the value of these models.

We have shown previously that complete loss of Ubc9, and hence of sumoylation, is early embryonic lethal in mice [10]. Similarly, acute loss of Ubc9 in adult mice leads to rapid death caused by degeneration of the intestinal epithelium [23]. In this setting, lack of sumoylation primarily affects the crypt compartment by causing the disappearance of the $\mathrm{Lgr5}^{+} \mathrm{CBC}$ cells that leads to rapid disorganization of the crypt-villus axis, thereby severely compromising the genesis, function, positioning and survival of all differentiated epithelial cells. Since sumoylation is essential for cell and animal viability, we analyzed here the role of the SUMO pathway in cancer-related processes using in vitro and in vivo Ubc9 haploinsufficient models. Loss of a single $U b c 9$ allele in transformed fibroblasts conferred enhanced proliferative and transformed phenotypes. In addition, while without any major phenotypic effects in mice in normal conditions, Ubc9 haploinsufficiency was found to enhance intestinal tumorigenesis and to provoke earlier death in Apc-deficient backgrounds. Furthermore, we found this phenotype to be associated with both increased numbers of crypt-based $\mathrm{Lgr}^{+} \mathrm{CBC}$ cells and exacerbated stemness, inflammatory and cancer-related gene expression programs in this cellular context. These results uncover an unexpected oncosuppressive role of sumoylation with potential impact for cancer treatment.

\section{Results}

\section{Ubc9 haploinsufficiency promotes cell growth and transformed phenotypes in vitro and ex vivo}

To address the role of Ubc9 in controlling cell proliferation and tumorigenesis, we used mice haploinsufficient for Ubc9 which harbour a floxed and a null $U b c 9$ allele $\left(U b c 9^{f /-}\right)$ [23]. While $U b c 9^{-/-}$animals are embryonic lethal, $U b c 9^{f /-}$, like $U b c 9^{+/-}$mice display no overt phenotype in normal conditions, apart from a slight $(10 \%)$ decrease in body size and body weight, when compared to wild-type (WT) littermates [10]. Murine embryo fibroblasts (MEFs) derived from a pool of three E12.5 WT or $U b c 9^{f /-}$ embryos were transformed by retroviral transduction of a dominant-negative (DN) p53 mutant together with the HRAS ${ }^{\mathrm{V} 12}$ oncogene. $U b c 9^{f /-}$ MEFs were found to grow significantly faster than $U b c 9^{+/+}$MEFs and inducible deletion of the remaining floxed $U b c 9$ allele by 4-hydroxytamoxifen (4-OHT) [23] expectedly abrogated their proliferation capacity (Fig. 1a). This pattern correlated with suppression of Ubc9 protein expression, reduction in global sumoylation and appearance of free SUMO1 and SUMO2/3 (hereafter SUMO2-) (Fig. 1b).

To further characterize the proliferative advantage of non-treated $U b c 9^{f /-}$ over $U b c 9^{+/+}$transformed MEFs, we analyzed cell cycle distribution and apoptosis rates at 4 days post-passage, when cells are still non-quiescent but the difference in cell numbers is clearly evident (Fig. S1a). While the cell cycle distribution was very similar for cells of both genotypes (Fig. S1b), we found a slightly larger 
A

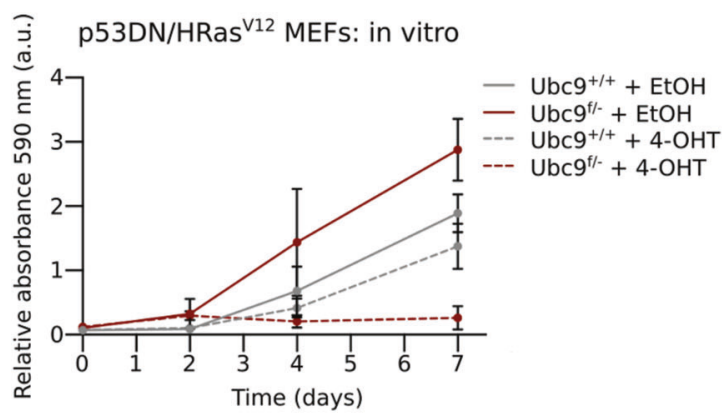

C

2D plate colony formation

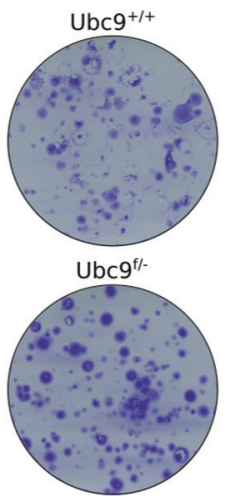

\section{$\mathbf{F}$}

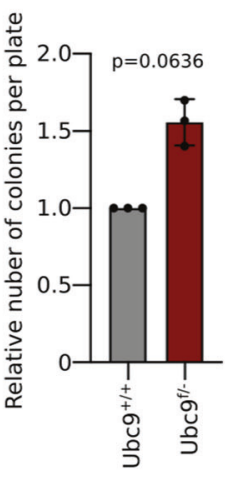

$\mathbf{F}$

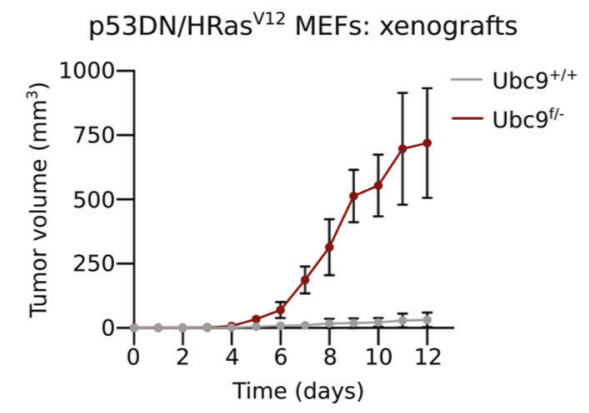

Fig. 1 Ubc9 haploinsufficiency promotes cell growth and malignant phenotypes in vitro. a Growth curve of $\mathrm{p} 53 \mathrm{DN} / \mathrm{HRAS}^{\mathrm{V} 12}$ transformed MEFs derived from 3 pooled embryos $U b c 9^{+/+}$and $\mathrm{Ubc}^{\mathrm{f} /-}$ Rosa26-Cre $e^{\text {ERT2 }}$ treated with $100 \mu \mathrm{g} / \mathrm{mL} 4-\mathrm{OHT}$ or EtOH (control). $590 \mathrm{~nm}$ absorbance of cell-trapped crystal violet was measured to estimate relative cell numbers. Mean $\pm \mathrm{SD}, n=3$ biological replicates. b Western-blotting showing SUMO1 and SUMO2 conjugates and Ubc9 expression in $\mathrm{Ubc}^{+/+}$and $\mathrm{Ubc} 9^{f /-} \mathrm{Rosa} 26-\mathrm{Cr} e^{E R T 2}$ MEFs treated with $100 \mu \mathrm{g} / \mathrm{mL}$ 4-OHT for 0,2 , 4, and 7 days. c Capacity of transformed MEFs for anchorage-dependent 2D plate colony formation. Representative images and quantification showing
B

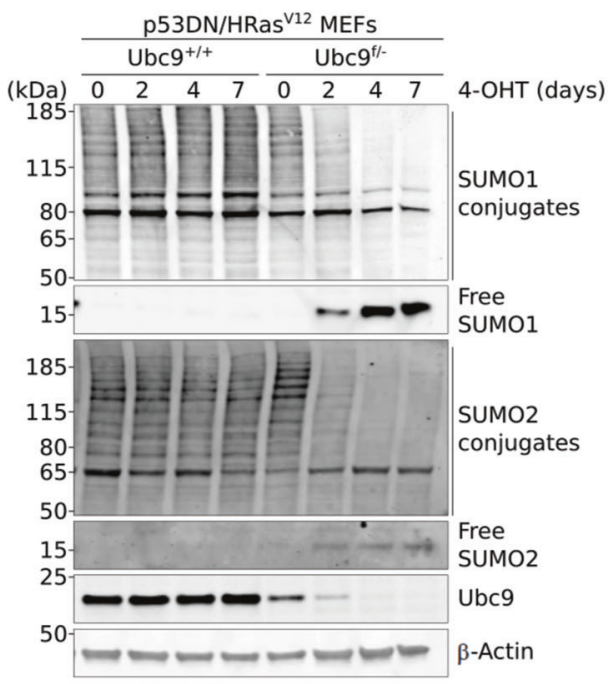

E oci formation

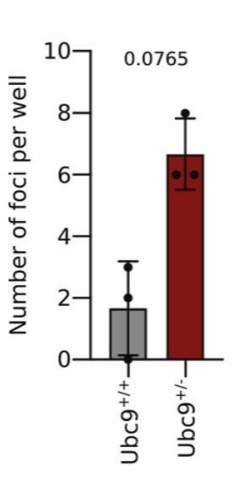

3D soft-agar colony formation

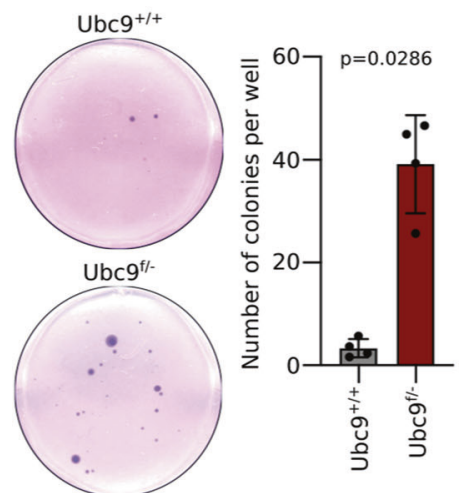

G

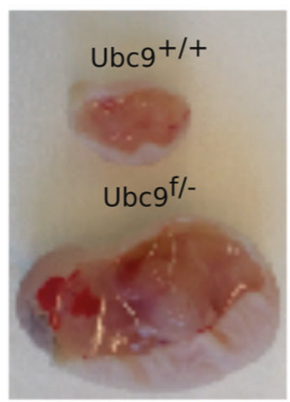

mean \pm SD. $n=3$ biological replicates in duplicates. Unpaired twotailed Mann-Whitney test was used. d Capacity of transformed MEFs for loss of contact inhibition and foci formation, as in (c), with $n=3$ biological replicates. e Capacity of transformed MEFs for anchorageindependent 3D soft-agar colony formation, as in (c), with $n=4$ biological replicates in triplicates. f Growth curve of p53DN/ HRAS $^{\text {V12 }}$-transformed MEF xenografts in mice. Mean $\pm \mathrm{SD}, n=6$ mice injected with $U b c 9^{f /-}$ and $U b c 9^{+/+}$transformed MEFs in opposite flanks of each animal. g Representative picture of tumors, as in (f), taken from the same mouse 12 days after inoculation. 
fraction of apoptotic cells in $\mathrm{Ubc}^{+/+}$(mean 9.8\%) compared to $U b c 9^{f /-}(6.7 \%)$ cells (Fig. S1c), a difference, however, likely insufficient to fully explain the observed, significant 1.7 -fold difference in cell numbers after 4 days' growth. To next see whether the growth difference could be related to the levels of the exogenous oncogenes, we compared the expression of p53 and HRAS between both genotypes. RT-qPCR analysis showed that levels of endogenous p53 and HRas are similar in both contexts (Fig. S1d). Interestingly, however, expression of exogenous murine $p 53 D N$ and human $H R A S^{V 12}$ mRNA was higher in $U b c 9^{+/+}$cells (Fig. S1e), a finding also confirmed for HRAS proteins by Western blot (Fig. S1f). Together, this suggests that the increased proliferation capacity displayed by mutant MEFs is not driven by higher oncogene activity but might rather be a consequence of a more transformationprone basal state in Ubc9 haploinsufficient MEFs.

The ability of transformed cells to form colonies of clonal origin on plates and in agar, or to form foci when seeded at high densities, is related to their capacity to grow and divide in an anchorage-dependent and anchorage-independent manner, or to lose contact inhibition, respectively [24-26]. Transformed $U b c 9^{f /-}$ MEFs formed colonies and foci more efficiently than their WT counterparts, as seen by the 1.6-, 4and 12 -fold growth increase in 2D plate colony-, foci formation and 3D soft-agar colony assays, respectively (Fig. $1 \mathrm{c}-\mathrm{e})$. To transpose these findings in vivo, we xenografted nude mice with transformed $U b c 9^{+/+}$and $U b c 9^{f /-}$ MEFs and found that Ubc9 haploinsufficiency greatly enhanced tumor growth, consistent with the findings obtained in vitro
(Fig. 1f-g). Altogether, these data suggest that mild Ubc9 downregulation confers a growth advantage consistent with the establishment of an exacerbated transformed state.

\section{Ubc9 haploinsufficiency favors polyp formation in an $\mathrm{Apc}^{\mathrm{f} /+}$ intestinal cancer mouse model}

We next investigated the effect of Ubc9 haploinsufficiency on tumor initiation and development in vivo, using the $A p c^{f /+}$ intestinal cancer mouse model. First, and in agreement with the situation in WT and $U b c 9^{f /-}$ MEFs (compare "0" lanes in Fig. 1b), we found that global profiles of SUMO-conjugated proteins in whole intestinal extracts from WT and $U b c 9^{+/-}$mice are indistinguishable (Fig. 2a), although this does not exclude that effects on specific substrates go undetected by this global analysis. Of note, the Ubc9 protein level was reduced to about a half of normal levels in $U b c 9^{f /-}$ intestines, indicating the absence of compensatory mechanisms for Ubc9 expression in these animals (Fig. 2a). Furthermore, anatomopathological analysis of intestines from mice of both genotypes did not reveal significant differences in the general architecture of crypts (Fig. S2a).

Next, we generated $U b c 9 ;$ Villin-Cre ${ }^{E R T 2} ; A p c^{f /+}$ mouse strains in which $\mathrm{Cr}^{\mathrm{ERT} 2}$ recombinase expression is driven by the intestine-specific Villin promoter [27, 28]. After Cre $\mathrm{CRT}^{\mathrm{ET} 2}$ activation by intraperitoneal injections of 4-OHT, the loxPflanked exon 14 in one allele of the Apc tumor suppressor is deleted all along the crypt-villus axis leading to the initial stage of the multi-step transformation process $[19,20]$ in
A

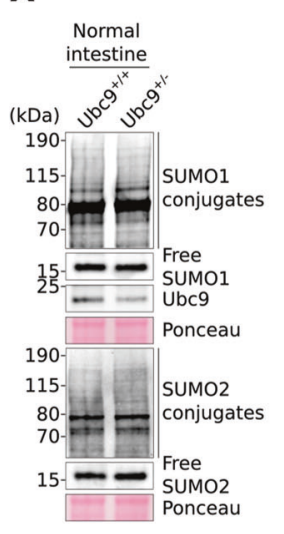

B

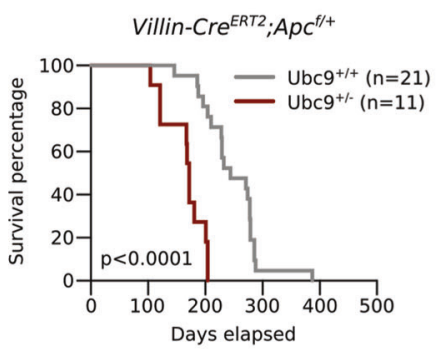

C

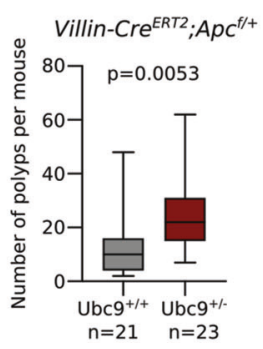

D

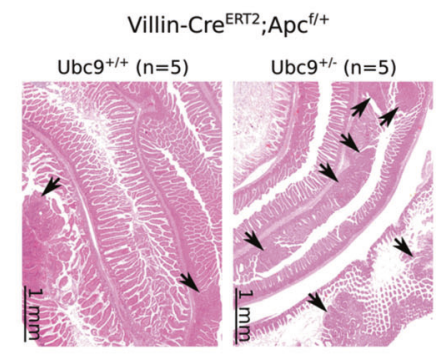

E

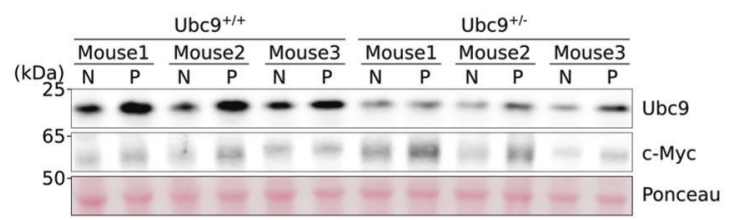

Fig. $2 U b c 9$ haploinsufficiency favors polyp formation in Villin$\mathrm{Cre}^{\text {ERT2 }} ; \mathrm{Apc}^{\mathrm{f} /+}$ intestinal cancer mouse model. a Western-blotting of SUMO1 and SUMO2 conjugates and Ubc9 in intestines from healthy mice. b Kaplan-Meier survival curves of $U b c 9^{+/+}$and $U b c 9^{+/-}$Villin$C r e^{E R T 2} ; A p c^{f /+}$ mice treated with $4-\mathrm{OHT}(0.1 \mathrm{mg}$ per $\mathrm{g}$ of body weight). Log-rank test was used. c Median with min, 25 and 75 percentiles, and max number of small intestinal adenomas $(\geq 0.5 \mathrm{~mm}$ in diameter) in $U b c 9^{+/+}$and $U b c 9^{+/-}$mice 12 weeks after treatment with 4-OHT as in (b). Unpaired two-tailed $t$ test was used. d Histological analysis of hematoxylin and eosin (H\&E)-stained small intestinal sections obtained as in (c). Arrows indicate lesions. e Western-blotting of Ubc9 and c-Myc in polyps (P) and normal (N) intestine samples obtained as in (c) in three different mice per group. 
either the WT or $U b c 9^{+/-}$genetic background. Surprisingly, we found that 4-OHT treatment resulted in shortened survival of $U b c 9^{+/-}$mice compared to their $U b c 9^{+/+}$counterparts (Fig. 2b). To determine whether this effect was related to tumor incidence and/or load, we assessed the number of adenomatous polyps $(\geq 0.5 \mathrm{~mm}$ in diameter) generated 12 weeks after 4-OHT treatment. We found that $U b c 9^{+/-}$ mice developed twofold more adenomas, with medians of 10 and 22 polyps per animal in small intestines of $U b c 9^{+/+}$and $U b c 9^{+/-}$mice, respectively (Figs. 2c, S2b). Hematoxylin and eosin (H\&E) staining of these samples further confirmed the increased frequency of both dysplasia and tumors in $U b c 9^{+/-}$ mice (Figs. 2d, S2c). No differences in the morphology and general organization of tumors and dysplasia foci were found (Figs. 2d, S2b-d). Finally, the median size of $U b c 9^{+/-}$polyps appears to be half that of WT (Fig. S2e), although, due to rounding off the measurements to the nearest $1 \mathrm{~mm}$ increment, the real median size difference may likely be less pronounced. A control experiment to address the possibility that Ubc9 haploinsufficiency merely accelerates Apc loss, and hence tumorigenesis, revealed that reduction of Ubc9 levels, here carried out in human U2OS cells, likely does not affect homologous recombination (HR), a process strictly required for tumorigenesis by loss of the second, WT Apc allele in the intestines of Ubc9; Villin-CreERT2;Apc ${ }^{f /+}$ mice (Fig. S2h-j). This suggests that the higher adenoma frequency seen in $U b c 9^{+/-}$mice is unlikely due to enhanced HR-dependent inactivation of the native $A p c$ allele. Together, these data indicate that decreasing Ubc9 levels to a half-dose promotes intestinal tumorigenesis. Moreover, they suggest that tumor initiation, rather than development, is enhanced in this process.

\section{$\mathrm{Ubc9}^{+/+}$and $\mathrm{Ubc9}^{+/-}$mice display similar transcriptomic profiles in adenomatous polyps or in normal intestinal tissue}

We next sought to pinpoint the molecular pathways responsible for the stimulated intestinal tumorigenesis observed in $U b c 9^{+-}$mice. For this, we first compared Ubc9 protein levels in normal $(\mathrm{N})$ and polyp $(\mathrm{P})$ intestinal samples from Villin-Cre ${ }^{E R T 2} ; \mathrm{Apc}^{f /+}$ mice 12 weeks after 4-OHT treatment. Higher c-Myc levels confirmed the transformed nature of polyp samples from both $U b c 9^{+/+}$and $U b c 9^{+/-}$genotypes (Fig. 2e). Besides the expected reduction in $U b c 9^{+/-}$samples, interestingly, Ubc9 levels were nevertheless higher in polyps than in adjacent normal tissue in both the WT and the $U b c 9^{+/-}$contexts (Fig. 2e).

Transcriptomic analysis of dissected polyps as well as of neighboring normal intestinal tissues from these animals revealed a total of only 11 differentially-expressed genes (DEGs, $\mid \log 2 \mathrm{FCl}>\log 2(1.5)$ and adj. $p$ value $(\mathrm{FDR})<0.05$ ), with 7 and 4 up- and downregulated, respectively, in
$U b c 9^{+/-}$polyps when compared to WT (Fig. S2f, Table S1). Similar analysis of the neighboring normal intestinal tissue yielded only a set of 31 DEGs, of which 8 and 23 were up- and downregulated, respectively, in the $U b c 9^{+/-}$ animals (Fig. S2g, Table S2). Gene Ontology (GO) analysis applied to these small lists of genes failed to discern any specific ontology term related to cancer development, suggesting that $U b c 9$ status has little or no effect on the specific gene expression signatures of polyps or normal intestinal tissue, or that analysis of these "bulk" tissues may have failed to unmask cell type-specific transcriptional differences.

\section{Ubc9 haploinsufficiency increases tumorigenesis upon Apc loss in $\mathrm{Lgr5}^{+}$CBC cells}

Given the enhancing effect of reduced Ubc9 levels on intestinal tumorigenesis, yet the absence of a clear transcriptomic effect in either bulk polyps or normal intestine, we focused subsequent analyses on the more restricted set of CBC cells that includes early progenitors, and importantly, the intestinal stem cells [22]. For this, we generated Ubc9;Lgr5-IRES$E G F P-C r e^{E R T 2} ; A p c^{f /+}$ mouse strains in which deletion of one loxP-flanked $A p c$ allele is restricted to CBC cells [21]. In line with previous results (Fig. 2b), $U b c 9^{+/-}$4-OHT-treated mice displayed shorter survival than their $\mathrm{Ubc}^{+/+}$counterparts (Figs. 3a, S3a). Moreover, mutant mice developed $>2$-fold more adenomas $(\geq 0.5 \mathrm{~mm}$ in diameter), albeit with smaller size, in the small intestine 16 weeks after 4-OHT treatment (Figs. 3b, S3b). It is noteworthy that transformation rates in Lgr5-IRES-EGFP-Cre ${ }^{E R T 2}$ mice were approximately threefold lower than previously seen in Ubc9; Villin-Cre $e^{E R T 2} ; A p c^{f / 4}$ animals, with medians of 2.5 and 6.5 polyps per mouse counted in $U b c 9^{+/+}$and $U b c^{+/-}$mice, respectively. This is also in line with the improved overall survival of $U b c 9 ; \operatorname{Lr} 5$ IRES-EGFP-Cre ${ }^{E R T 2} ; A p c^{f /+}$ compared to Ubc9; Villin-Cre ${ }^{E R T 2}$; $A p c^{f /+}$ mice (compare Figs. 2b, 3a). Histological analysis again revealed no differences in morphology or general organization of lesions attributable to Ubc9 status (Fig. 3c). Altogether, these data show that loss of one $U b c 9$ allele also enhances tumor formation in vivo in an Apc-null background limited to CBC cells.

\section{Transcriptomic analysis of $\mathrm{Lgr}^{+} \mathrm{CBC}$ cells reveals a cancer-related and pro-inflammatory basal state under Ubc9 haploinsufficiency}

Taking advantage of $L g r 5$-driven expression of EGFP in Lgr5-IRES-EGFP-Cre $e^{E R T 2}$ mice, we obtained fluorescenceactivated cell sorting (FACS)-purified Lgr5-EGFP $^{+}$cells derived from normal $\left(\mathrm{Apc}^{+/+}\right)$intestines from both $\mathrm{Ubc} 9^{+/+}$ and $U b c 9^{+/-}$mice and performed a comparative analysis of their transcriptomic profiles (Fig. S4a). We found 205 DEGs 
A

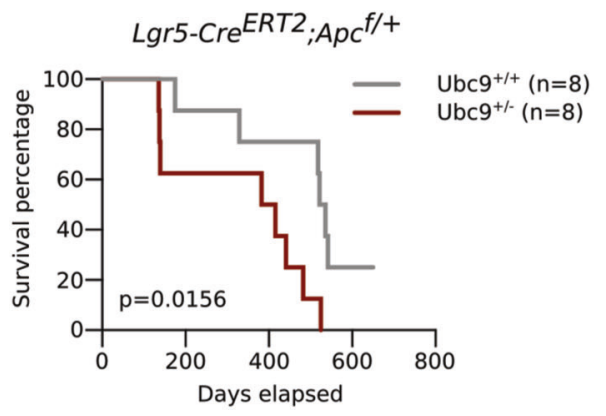

B

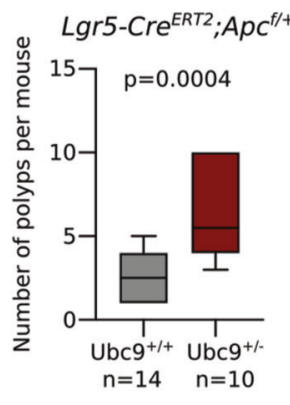

C

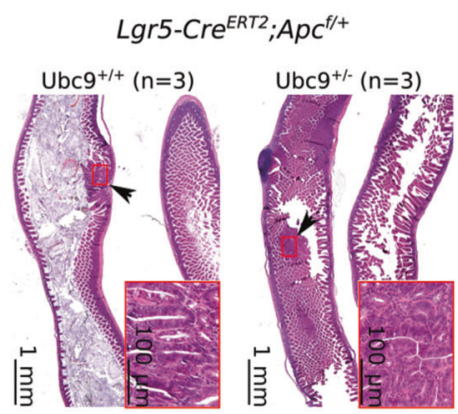

Fig. $3 U b c 9$ haploinsufficiency favors polyp formation in Lgr5IRES-EGFP-Cre ${ }^{E R T 2} ;$ Apc $^{f /+}$ intestinal cancer mouse model. a Kaplan-Meier survival curves of $U b c 9^{+/+}$and $U b c 9^{+/-}$Lgr5-IRES$E G F P-C r e^{E R T 2} ; A p c^{f /+}$ mice treated with $4-\mathrm{OHT}$ as in Fig. 1b. Logrank test was used. b Median number of small intestinal adenomas ( $\geq 0.5 \mathrm{~mm}$ in diameter) with min, 25 and 75 percentiles, and max values in $U b c 9^{+/+}$and $U b c 9^{+/-}$mice 16 weeks after treatment with 4-OHT, as in Fig. 1a. Unpaired two-tailed Mann-Whitney test was used. c Histological analysis of H\&E-stained small intestinal sections obtained as in (b). Arrows indicate lesions also shown in the magnified insets.
A Purified CBC cells: Lgr5-EGFP

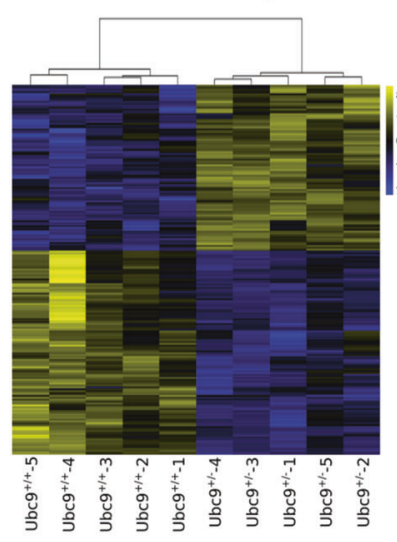

B

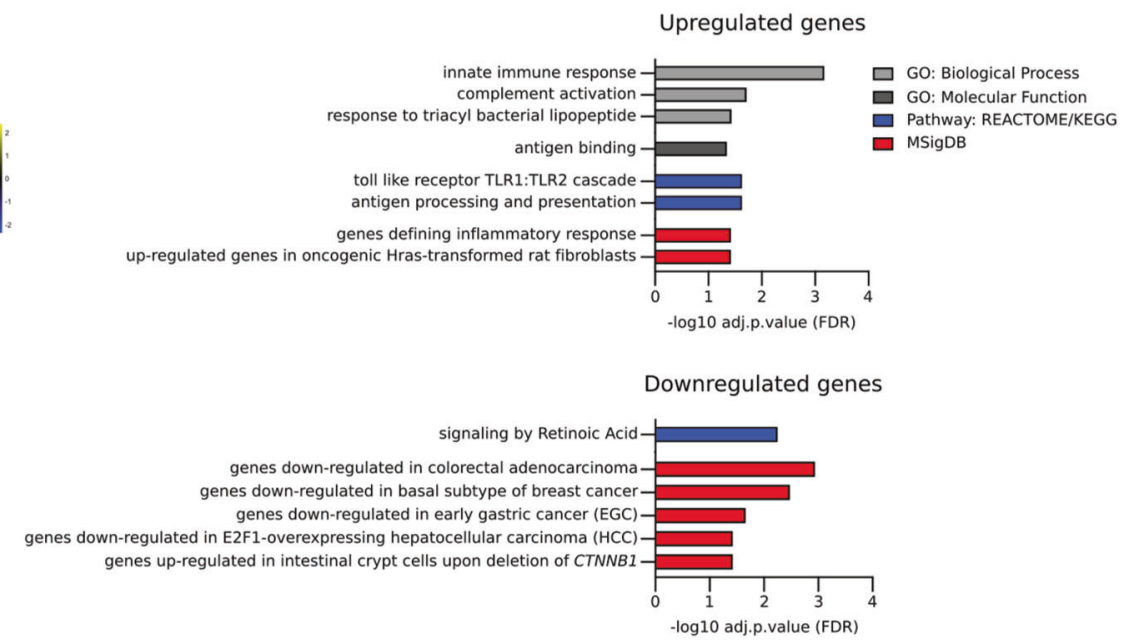

Fig. $4 \mathrm{Ubc}^{+/-} \mathrm{CBC}$ cells exhibit inflammatory and cancer-related transcriptomic signatures. a Heatmap of 205 differentially-expressed genes $(\log 2 \mathrm{FCl}>\log 2(1.5)$ and adj. $p$ value $(\mathrm{FDR})<0.05)$ in $\mathrm{Ubc} 9^{+/-}$ Lgr5-EGFP $^{+}$CBC cells from intestines of healthy Lgr5-IRES-EGFP$C r e^{E R T 2}$ mice purified by FACS. $n=5$ per group. b Gene Ontology

$(\log 2 \mathrm{FCl}>\log 2(1.5)$ and adj. $p$ value $(\mathrm{FDR})<0.05)$ in $U b c 9^{+/-}$Lgr5-EGFP $^{+}$cells, when compared to $U b c 9^{+/+}$ Lgr5-EGFP $^{+}$cells, of which 92 and 113 were up- and downregulated, respectively (Fig. 4, Table S3).

Interrogation of curated gene expression databases, using as template up- and downregulated genes separately, now revealed a group of DEGs that have been associated with different types of cancers. Among the upregulated genes were genes reported to have increased expression in HRastransformed rat fibroblasts (Fig. 4b, Tables S3, S4). Among downregulated DEGs in $\mathrm{Ubc}^{+/-} \mathrm{Lgr}^{-\mathrm{EGFP}^{+}}$cells, up to $17 \%$ were genes found to be repressed in early gastric cancer,
(GO) and Pathway analysis of differentially-expressed genes upregulated (upper) and downregulated (lower) in Ubc9 $9^{+/}$Lgr5-EGFP $^{+}$ CBC cells with adjusted $p$ value (FDR Benjamini-Hochberg) $<0.05$. GO's Biological Process, and pathways from KEGG and REACTOME and MSigDB databases were challenged.

colorectal adenocarcinoma, E2F1-overexpressing hepatocellular carcinoma and a subtype of basal breast cancer. Significantly, some downregulated genes (Capn9, Pnliprpl, Pnliprp2, Cgref1, Cyp2ul, Aldhla1, and Car4) had also been found previously [29] to be upregulated in intestinal crypts upon deletion of $\beta$-catenin (CTNNB1; Fig. 4b, Tables S3, S4).

A further significant feature suggested by GO analysis of genes upregulated in $U b c 9^{+/-}$Lgr5-EGFP ${ }^{+}$cells were tophit signatures for the inflammatory response, innate immune response, genes involved in the response mediated by the membrane-bound toll-like receptors, complement activation and response to bacteria (Fig. 4b, Table S4). Genes from the 
above-mentioned signatures represent $25 \%$ of the upregulated DEGs found in $U b c 9^{+/-}$Lgr5-EGFP ${ }^{+}$cells (Table S3), indicating that loss of one Ubc9 allele triggers a proinflammatory state in $\mathrm{Lgr}^{+} \mathrm{CBC}$ cells.

\section{Ubc9 $^{+/-}$crypts harbor more Lgr5 ${ }^{+}$CBC cells}

We next assessed whether Ubc9 haploinsufficiency could be associated with changes in intestinal crypts in Lgr5-IRES$E G F P-C r e^{E R T 2}$ mice. In order to evaluate the localization of both $\mathrm{Lgr}^{+}$and Paneth cells, intestines from $U b c 9^{+/+}$and $U b c 9^{+/-}$mice were prepared for confocal microscopy and analyzed for EGFP and lysozyme, respectively. No differences were detected in the general architecture or localization of Lgr5-EGFP ${ }^{+}$cells and Paneth cells within crypts from mice of both genotypes (Figs. 5a, S2a). Strikingly, blinded counting, however, revealed an elevated number of small intestinal Lgr5-EGFP ${ }^{+} \mathrm{CBC}$ cells in $\mathrm{Ubc}^{+/-}$crypts (medians of 21 and 24 per crypt for $U b c 9^{+/+}$and $U b c 9^{+/-}$, respectively; Fig. 5a, b). This trend was consistent all along the small intestine but was not seen in colonic crypts (Fig. S5a, b). Thus, Ubc9 haploinsufficiency leads to a modest (15\%) but significant expansion of the cell compartment containing intestinal stem cells. In addition, comparison of our transcriptomic data with a previously identified $\mathrm{Lgr}^{+}$ stem cell signature gene set [30] revealed a significant enrichment of the signature genes in the Ubc $9^{+/-}$CBC cells, suggesting a higher stemness state in mutant cells compared to the WT (Fig. 5c, Table S5).

\section{Enhanced sumoylation in intestinal crypts and polyps}

Finally, we analyzed the sumoylation status in partiallypurified villi and crypt-based cells from $U b c 9^{+/+}$and $U b c 9^{+/-}$ animals. For this, washed, longitudinally-opened intestines were first scraped to release villi cells and then treated with EDTA to liberate crypt-based cells for analysis by western blotting. As expected, expression of markers for undifferentiated cells, c-Myc [31], and differentiated cells, Hoxa5 [32], was higher and lower, respectively, in crypt-enriched compared to villi-containing samples (Fig. 5d). In line with this observation, mRNA expression of $\beta$-catenin/Wnt pathway components known to be highly expressed in crypts, such as c-Myc, Smoc2 and Axin2 [30] was also found to be elevated (Fig. S5c, "C"), whereas expression of the differentiation marker Krt20 [33] was found to be higher in villienriched samples (Fig. S5c, "V"), thus also validating the proper separation of cell populations. Significantly, total free SUMO and SUMO conjugate levels were higher in cryptenriched cell fraction (Fig. 5d, "C"), with, however, no clear differences between $U b c 9^{+/+}$and $U b c 9^{+/-}$samples, as also seen in whole intestine (Fig. 2a). Higher Ubc9 (see also
Fig. 2e) and free SUMO levels were similarly observed in adenomatous polyps $(\mathrm{P})$ when compared to normal tissue $(\mathrm{N})$ in both $U b c 9^{+/+}$and $U b c 9^{+/-}$mice in an Apc-loss background (Fig. S5d). Remarkably, while this was accompanied by increased levels of SUMO conjugates in polyps from WT mice, we failed to detect such globally enhanced sumoylation in the tumors from $U b c 9^{+/-}$mice, indicating that, in the latter, Ubc9 levels appear limiting for efficient sumoylation. Together, these results point towards a greater global SUMO pathway activity in both the proliferative crypt compartment and in the dysplastic polyp tissue. Of note, this is consistent with findings in human colon adenocarcinomas that also exhibit elevated Ubc9 protein expression [34] and almost twofold increases in median transcript levels in tumors $(n=275)$ compared to non-tumor $(n=349)$ tissues (GEPIA database, http://gepia.cancer-pku.cn/index.html, queried for UBE2I (Ubc9)) [35].

\section{Discussion}

Here we have shown that Ubc9 haploinsufficiency enhances tumorigenesis and lethality in Apc loss-driven mouse models of intestinal neoplasia. This surprising outcome is associated with a moderate but detectable (15\%) increase in the number of $\mathrm{Lgr5}^{+} \mathrm{CBC}$ cells, which, in addition, display a higher degree of stemness and cancer-, inflammation- and innate immunity-related gene expression signatures. We also found that Ubc9 haploinsufficiency enhances the proliferative capacities of transformed MEFs both in vitro and ex vivo. Together, these studies establish an as yet unappreciated tumor-suppressive activity for sumoylation.

A basic question emerging from these results is whether Ubc9 haploinsufficiency indeed translates into reduced cellular sumoylation, given that globally visible SUMO conjugate levels appear largely unaffected by loss of one $U b c 9$ allele, notably in transformed MEFs and healthy intestine (Figs. 1b, 2a). It is difficult to imagine, however, that the observed phenotypes under haploinsufficiency result from compromised sumoylation-independent Ubc9 activity. Rather, we suggest the involvement of specific "under-modified" substrate conjugates, whose detection is masked here by other, abundantly-modified proteins in such global analysis. The identification of these specific substrates, although technically challenging, may thus shed important light on the pathways and molecular mechanisms involved. Moreover, while Ubc9 haploinsufficiency does not always reduce sumoylation visibly, it does reduce sumoylation capacity, as seen most clearly in polyps, a tumor state highly demanding in sumoylation (Fig. S5d). This indicates that different cell types make different demands on the SUMO system and, conversely, that they might therefore display different sensitivities to its reduction. The smaller polyp size under Ubc9 
A
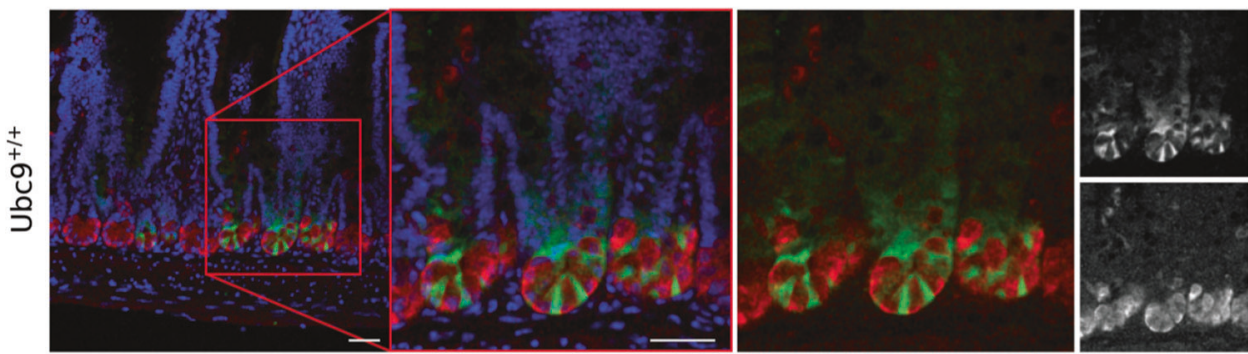

EGFP

(Lgr5 ${ }^{+} \mathrm{CBC}$ cells $)$
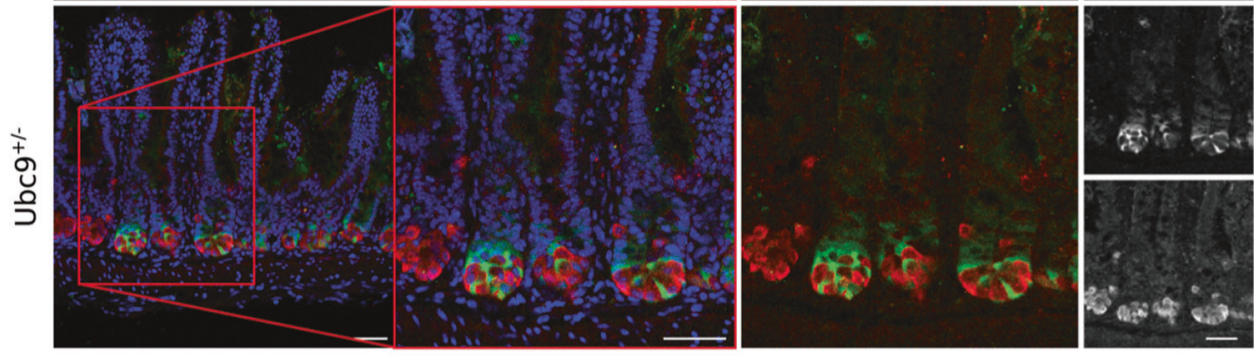

Lysozyme

(Paneth cells)

DAPI $\quad \operatorname{EGFP}\left(\mathrm{Lgr5}^{+} \mathrm{CBC}\right.$ cells $)$

Lysozyme (Paneth cells)

\section{B}

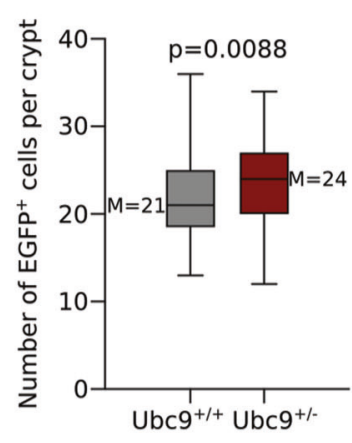

C

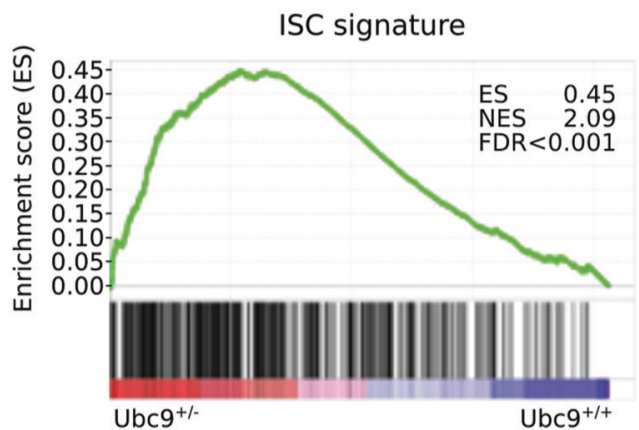

D

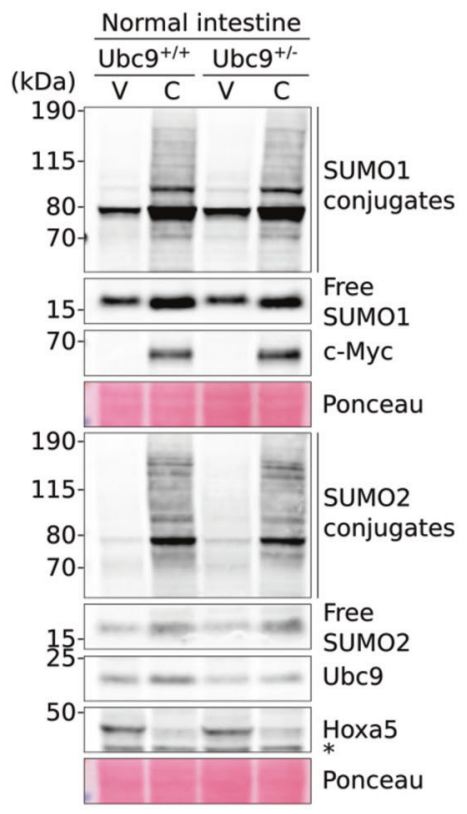

Fig. $5 \mathrm{Ubc}^{+/-}$crypts harbor more $\mathrm{Lgr5}^{+} \mathrm{CBC}$ cells. a Confocal images of small intestinal crypts $U b c 9^{+/+} ;$Lgr5-IRES-EGFP-Cre $e^{E R T 2}$ and $U b c 9^{+/-} ;$L gr5-IRES-EGFP-Cre $e^{E R T 2}$ mice in an $A p c^{+/+}$background. EGFP ( $\mathrm{Lgr}^{+} \mathrm{CBC}$ cells, green), Lysozyme (Paneth Cells, red), and DAPI are shown. Scale bars, $50 \mu \mathrm{m}$. b Median (M, shown on graph) with min, 25 and 75 percentiles, and max values of Lgr5$\mathrm{EGFP}^{+} \mathrm{CBC}$ cells per crypt in small intestines as detected in (a). $n=$ 137 crypts, 5 mice and 127 crypts, 5 mice for Ubc $9^{+/+}$and $\mathrm{Ubc} 9^{+/-}$,

haploinsufficiency (Figs. S2e, S3b) indeed suggests that insufficient sumoylation capacity may be a handicap for tumor growth.

respectively. Unpaired two-tailed t test was used. c Significant enrichment of ISC signature genes in $\mathrm{Ubc}^{+/-}$FACS-purified $\mathrm{Lgr}^{+}$-GFP CBC cells analyzed by gene set enrichment analysis (GSEA). ES enrichment score, NES normalized enrichment score. d Western-blotting of SUMO1 and SUMO2 conjugates, Ubc9, c-Myc and Hoxa5 in intestinal villi (V)- and crypt (C)-enriched populations from healthy Ubc9; Lgr5-IRES-EGFP-Cre ${ }^{E R T 2}$ mice. *Non-specific.

Why, in contrast, Ubc9 haploinsufficiency in transformed MEFs results in enhanced proliferation (Fig. 1) could be explained by both the reduction of an as yet 
uncharacterized growth-inhibiting function of sumoylation, that may also apply to $\mathrm{Lgr} 5^{+} \mathrm{CBC}$ cells (Fig. 5a, b), and to a reduced sensitivity to inhibition of these different cell types. In both cases our results show that reduced sumoylation capacity, when mild, is not necessarily associated with reduced proliferative capacity.

Our findings that $U b c 9^{+/-} \operatorname{Lgr}^{+} \mathrm{CBC}$ cells display a pro-inflammatory state under normal conditions (Fig. 4b and Tables S3, S4) are in line with sumoylation acting as a general repressor of the inflammatory response, as shown in myeloid cells [36]. Inhibition of Ubc9 has been shown to activate pro-inflammatory regulators, such as RelA, cFos, cJun, and IFN- $\gamma$ in cultured HCT- 8 epithelial cells, and to lead to decreased amounts of the anti-inflammatory IL-10 cytokine in primary intestinal epithelial cells [37]. Lower sumoylation rates, possibly due to enhanced SENP7 desumoylase activity, were also detected in patients with inflammatory bowel disease and were described as a prerequisite for the onset of inflammation in colons from mice with dextran sodium sulfate- (DSS-) induced colitis [37, 38]. Significantly, previous studies also indicate a link between increased CBC cell numbers, as seen here, and inflammation. For example, a small increase in the number of $\mathrm{Lgr}^{+} \mathrm{CBC}$ cells and a significant rise in $\mathrm{BrdU}^{+}$proliferating cells per crypt in the large intestine has been described in mice administrated with the inflammatory agent DSS [39]. Similarly, intestinal organoids treated with low doses of pro-inflammatory IL-22 [40] or the chronic colitis-associated cytokine TNF$\alpha$ [39], displayed a small but significant increase in the percentage of $\mathrm{Lgr5}^{+} \mathrm{CBC}$ cells. These observations indicate that mild and non-pathogenic levels of inflammation may indeed promote CBC cell renewal, consistent with our results (Figs. 5a, b, S5a) and the known roles of inflammation in tumorigenesis and tissue regeneration $[41,42]$. Conditions, such as Ubc9 haploinsufficiency that increases the number of stem cells together with promoting a pro-inflammatory state, would thereby also increase the number of cells with tumor-initiating potential, and hence tumorigenesis.

Recent findings showing that sumoylation represents a significant barrier to cell fate change $[43,44]$ may provide a further explanation for increased CBC cell number (Figs. 5a, b, S5a). While CBC cells dividing symmetrically at the crypt base represent the normal source of intestinal stem cells for tissue maintenance, mounting evidence indicates that during injury, lost stem cells in the crypt can be replenished from the " +4 " "reserve" cell population [45], from committed secretory progenitor cells [46], from Paneth and enteroendocrine lineage precursors [47, 48] or from early enterocyte lineage (absorptive) cell precursors [49]. It would thus be interesting to determine if Ubc9 haploinsufficiency, like injury, increases such cellular plasticity leading to increased $\mathrm{CBC}$ cell number. Moreover, in this scenario, this may also contribute to a second function, enhanced inflammatory signaling, shown necessary for the conversion of such non-stem cells to cells with tumorinitiating potential [50].

Finally, our finding of a tumor-suppressive function of sumoylation appears at odds with previously established roles of sumoylation in cancer. Indeed, the pro-tumorigenic role of sumoylation is inferred from the enhanced levels of SUMO pathway enzymes, and hence, sumoylation dynamics, and from the accrued sensitivity to inhibition of global sumoylation seen in many cancer cells [reviewed in ref. 7]. It must be borne in mind, however, that, as shown here, Ubc9 haploinsufficiency increases the frequency of tumor initiation events, as seen by the increased number of polyps. Indeed, under normal $\left(A p c^{+/+}\right)$conditions, reduced (i.e., haploinsufficient) Ubc9 activity appears sufficient to ensure cell functionality. However, when cells face stress, such as loss of Apc tumor suppressor activity, this may become a handicap for maintaining the controlled nonmalignant state, in line also with the observed cancer-related transcriptome signature.

Together, our findings support a view of sumoylation as a buffering system against stress. As such, this system must first be overcome (aided experimentally by Ubc9 haploinsufficiency) during the initiation phase of tumorigenesis, to be then rewired, or even enhanced, to serve in mechanisms that ultimately protect the cancerous state. Given the "druggability" of the SUMO pathway [16], deeper understanding of these dose- and cell type dependent, seemingly paradoxical consequences of targeting the SUMO system may have important therapeutic implications.

\section{Materials and methods}

\section{Mice}

$U b c 9^{+/-}[10]$ and $U b c 9^{f /-} ; \operatorname{Ros} a 26 C r e^{E R T 2}$ [23] mice have been described previously. $U b c 9^{+/-}$;Villin-Cre $e^{E R T 2} ; A p c^{f /+}$ mice were obtained by crossing $U b c 9^{+/-}$with Villin-Cre $e^{E R T 2}$ mice [28] and then with $A p c^{\mathrm{f} /+}$ mice [27]. Ubc $9^{+/-} ;$Lgr5-IRES-EGFP$C r e^{E R T 2}$ animals were obtained by crossing $U b c 9^{+/-}$with Lgr5-IRES-EGFP-Cre $e^{E R T 2}$ mice [21]. Ubc9 $9^{+/-} ;$Lgr5-IRES$E G F P-C r e^{E R T 2} ; A p c^{f /+}$ mice were created by crossing $U b c 9^{+/-}$; Lgr5-IRES-EGFP-Cre $e^{E R T 2}$ with $A p c^{f /+}$ mice [27]. See Supplementary Information for details.

\section{Histology}

Paraffin-embedded $4 \mu \mathrm{m}$ sections were stained using H\&E solution and blindly analyzed by a pathologist. See Supplementary Information for details. 


\section{Cell culture and growth of transformed MEFs}

MEFs were obtained from three $U b c 9^{f /-} ; \operatorname{Ros} a 26 \mathrm{Cr} e^{E R T 2}$ or $\mathrm{Ubc} 9^{+/+} ;$Rosa26Cre ${ }^{E R T 2}$ embryos at day E12.5 and pooled. Low-passage Rosa26-Cre $e^{E R T 2} \mathrm{p} 53 \mathrm{DN}$ and $\mathrm{HRAS}^{\mathrm{V} 12}$-transformed MEFs were treated with 4-hydroxytamoxifen (4OHT; Sigma-Aldrich) or ethanol (EtOH) as control for the indicated time for growth experiments and were also used for 2D plate colony formation (low density), foci formation (high density), and 3D soft-agar colony formation assays. See Supplementary Information for details.

\section{Homologous recombination reporter assay}

HR was determined as described previously [51] using a DRGFP reporter stably integrated into U2OS human osteosarcoma cells. See Supplementary Information for details.

\section{Western blotting}

Protein extracts from cultured cells were obtained by lysing the cells directly in Laemmli buffer (Bio-Rad, Hercules CA, USA). Protein extracts from tissues were obtained by immersing the tissue in RIPA buffer in Lysing Matrix Dcontaining tubes (MP Biomedicals, Illkirch, France). See Supplementary Information for details.

\section{Flow cytometry and fluorescence-activated cell sorting (FACS)}

Analysis of cell cycle and apoptosis in transformed MEFs was carried out using the Muse Cell Analyzer (Millipore, Hayward CA, USA) and the Muse Cell Cycle and Muse Annexin $\mathrm{V}$ and Dead Cell kits, respectively (Luminex, Austin TX, USA). Purification of $L g r 5-E G F P^{+} \mathrm{CBC}$ cells from normal non-treated Ubc9;Lgr5-IRES-EGFP-Cre ${ }^{E R T 2}$ mice and preparation of villi- and crypt-enriched populations were performed adapting previously-described protocols [30, 52]. Single EGFP ${ }^{\text {high }}, \mathrm{CD} 24^{\text {middle }}$, EpCam ${ }^{\text {positive }}$, and $\mathrm{PI}^{\text {negative }} \mathrm{CBC}$ cells were sorted using MoFlo Astrios Cell Sorter (Beckman Coulter). See Supplementary Information for details.

\section{Quantitative PCR}

RNA was purified using Trizol (Molecular Research Center, Cincinnati OH, USA) according to manufacturer's recommendations. Quantitative real-time PCR was performed using the primer sets specified in Supplementary Table S6.

\section{Transcriptome profiling}

RNA profiling was performed using GeneChip MoGene 2.0 ST Array (Applied Biosystems) and normalized and analyzed using open-source Bioconductor packages on $\mathrm{R}$ [53, 54]. Differentially-expressed genes (adj. $p$ value (FDR) $<0.05$ and $\mid \log 2 \mathrm{FCl}>1.5)$ were used to challenge curated databases [55-61]. For GSEA analysis, all differentially and non-differentially-expressed genes in FACS-purified EGF$\mathrm{P}^{\text {high }} \mathrm{CBC}$ cells were queried against a previously published intestinal stem cell signature [30]. See Supplementary Information for details.

\section{Detection of Lgr5-EGFP ${ }^{+}$CBC cells}

Cryo-sections (50 $\mu \mathrm{m}$ thick) of intestines from Ubc9; $\operatorname{Lgr} 5$ EGFP-IRES-Cre $e^{E R T 2}$ were analyzed for EGFP and Lysozyme expression by confocal microscopy and blindly analyzed using Fiji (ImageJ). See Supplementary Information for details.

\section{Statistics}

Cell-based experiments were carried out with at least three biological replicates. Experiments involving mice were performed in at least six animals per group. Statistical analysis was performed with GraphPad Prism 8 software. See Supplementary Information for details.

\section{Data availability}

Transcriptomic data from this study were deposited in the GEO database (https://www.ncbi.nlm.nih.gov/geo/) under accession numbers GSE146106 (Fig. 4) and GSE146039 (Fig. S2).

\section{Code availability}

Computer code can be accessed upon request.

Acknowledgements This work was supported by grants from INCa, LNCC (Equipe labellisée), Odyssey, ANR and ERC-AdG "SUMOSTRESS" and "SUMiDENTITY" to AD, and by the Josef Steiner Cancer Foundation and ERC-CoG "Cancer Recurrence" to JvR. IL was supported by Fondation ARC (PDF20170505624). We thank Dr. Giulia Nigro and the Institut Pasteur UTechS facility for technical input and Drs. Bob Weinberg, Maria Jasin, and Sérgio F. de Almeida for providing reagents.

Author contributions IL, EC, and AD planned the studies. IL and EC performed most of the experiments and analyzed the data. SIJE analyzed the $\mathrm{Lgr}^{+} \mathrm{CBC}$ cell content by immunofluorescence and interpreted the data with JvR. AA contributed to the mouse studies. P-FR and JPC performed the transcriptomic analysis, GJ the histological analysis. IL, J-SS and AD analyzed data and wrote the paper with input from JvR and SIJE.

\section{Compliance with ethical standards}

Conflict of interest The authors declare that they have no conflict of interest. 
Ethical approval Mice were bred at the Pasteur Institute animal facility in specific-pathogen-free conditions and studies were conducted in accordance with institutional guidelines and French and European law (Institut Pasteur animal experimentation ethics committee approval number 2016-0025).

Publisher's note Springer Nature remains neutral with regard to jurisdictional claims in published maps and institutional affiliations.

Open Access This article is licensed under a Creative Commons Attribution 4.0 International License, which permits use, sharing, adaptation, distribution and reproduction in any medium or format, as long as you give appropriate credit to the original author(s) and the source, provide a link to the Creative Commons license, and indicate if changes were made. The images or other third party material in this article are included in the article's Creative Commons license, unless indicated otherwise in a credit line to the material. If material is not included in the article's Creative Commons license and your intended use is not permitted by statutory regulation or exceeds the permitted use, you will need to obtain permission directly from the copyright holder. To view a copy of this license, visit http://creativecommons. org/licenses/by/4.0/.

\section{References}

1. Flotho A, Melchior F. Sumoylation: a regulatory protein modification in health and disease. Annu Rev Biochem. 2013;82:357-85.

2. Hendriks IA, Vertegaal AC. A comprehensive compilation of SUMO proteomics. Nat Rev Mol Cell Biol. 2016;17:581-95.

3. Golebiowski F, Matic I, Tatham MH, Cole C, Yin Y, Nakamura A, et al. System-wide changes to SUMO modifications in response to heat shock. Sci Signal. 2009;2:ra24.

4. Stankovic-Valentin N, Melchior F. Control of SUMO and Ubiquitin by ROS: signaling and disease implications. Mol Asp Med. 2018;63:3-17.

5. Jackson SP, Durocher D. Regulation of DNA damage responses by ubiquitin and SUMO. Mol Cell. 2013;49:795-807.

6. Bettermann K, Benesch M, Weis S, Haybaeck J. SUMOylation in carcinogenesis. Cancer Lett. 2012;316:113-25.

7. Seeler JS, Dejean A. SUMO and the robustness of cancer. Nat Rev Cancer. 2017;17:184-97.

8. Seufert W, Futcher B, Jentsch S. Role of a ubiquitin-conjugating enzyme in degradation of S- and M-phase cyclins. Nature. 1995;373:78-81.

9. Hayashi T, Seki M, Maeda D, Wang W, Kawabe Y, Seki T, et al. Ubc9 is essential for viability of higher eukaryotic cells. Exp Cell Res. 2002;280:212-21.

10. Nacerddine K, Lehembre F, Bhaumik M, Artus J, CohenTannoudji M, Babinet C, et al. The SUMO pathway is essential for nuclear integrity and chromosome segregation in mice. Dev Cell. 2005;9:769-79.

11. Neyret-Kahn H, Benhamed M, Ye T, Le Gras S, Cossec JC, Lapaquette P, et al. Sumoylation at chromatin governs coordinated repression of a transcriptional program essential for cell growth and proliferation. Genome Res. 2013;23:1563-79.

12. Luo J, Solimini NL, Elledge SJ. Principles of cancer therapy: oncogene and non-oncogene addiction. Cell. 2009;136:823-37.

13. Nagel R, Semenova EA, Berns A. Drugging the addict: nononcogene addiction as a target for cancer therapy. EMBO Rep. 2016;17:1516-31.

14. Kessler JD, Kahle KT, Sun T, Meerbrey KL, Schlabach MR, Schmitt EM, et al. A SUMOylation-dependent transcriptional subprogram is required for Myc-driven tumorigenesis. Science. 2012;335:348-53.
15. Yu B, Swatkoski S, Holly A, Lee LC, Giroux V, Lee CS, et al. Oncogenesis driven by the Ras/Raf pathway requires the SUMO E2 ligase Ubc9. Proc Natl Acad Sci USA. 2015;112:E1724-33.

16. He X, Riceberg J, Soucy T, Koenig E, Minissale J, Gallery M, et al. Probing the roles of SUMOylation in cancer cell biology by using a selective SAE inhibitor. Nat Chem Biol. 2017;13:1164-71.

17. Clevers $H$. The intestinal crypt, a prototype stem cell compartment. Cell. 2013;154:274-84.

18. Kinzler KW, Vogelstein B. Lessons from hereditary colorectal cancer. Cell. 1996;87:159-70.

19. Pino MS, Chung DC. The chromosomal instability pathway in colon cancer. Gastroenterology. 2010;138:2059-72.

20. Jackstadt R, Sansom OJ. Mouse models of intestinal cancer. J Pathol. 2016;238:141-51.

21. Barker N, van Es JH, Kuipers J, Kujala P, van den Born M, Cozijnsen $\mathrm{M}$, et al. Identification of stem cells in small intestine and colon by marker gene Lgr5. Nature. 2007;449:1003-7.

22. Barker N, Ridgway RA, van Es JH, van de Wetering M, Begthel $\mathrm{H}$, van den Born $\mathrm{M}$, et al. Crypt stem cells as the cells-of-origin of intestinal cancer. Nature. 2009;457:608-11.

23. Demarque MD, Nacerddine $K$, Neyret-Kahn H, Andrieux A, Danenberg E, Jouvion G, et al. Sumoylation by Ubc9 regulates the stem cell compartment and structure and function of the intestinal epithelium in mice. Gastroenterology. 2011;140:286-96.

24. Alvarez A, Barisone GA, Diaz E. Focus formation: a cell-based assay to determine the oncogenic potential of a gene. J Vis Exp. 2014. https://doi.org/10.3791/51742.

25. Borowicz S, Van Scoyk M, Avasarala S, Karuppusamy Rathinam MK, Tauler J, Bikkavilli RK, et al. The soft agar colony formation assay. J Vis Exp. 2014. https://doi.org/10.3791/51998.

26. Rafehi H, Orlowski C, Georgiadis GT, Ververis K, El-Osta A, Karagiannis TC. Clonogenic assay: adherent cells. J Vis Exp. 2011. https://doi.org/10.3791/2573.

27. Colnot S, Decaens T, Niwa-Kawakita M, Godard C, Hamard G, Kahn A, et al. Liver-targeted disruption of Apc in mice activates beta-catenin signaling and leads to hepatocellular carcinomas. Proc Natl Acad Sci USA. 2004;101:17216-21.

28. el Marjou F, Janssen KP, Chang BH, Li M, Hindie V, Chan L, et al. Tissue-specific and inducible Cre-mediated recombination in the gut epithelium. Genesis. 2004;39:186-93.

29. Fevr T, Robine S, Louvard D, Huelsken J. Wnt/beta-catenin is essential for intestinal homeostasis and maintenance of intestinal stem cells. Mol Cell Biol. 2007;27:7551-9.

30. Muñoz J, Stange DE, Schepers AG, van de Wetering M, Koo BK, Itzkovitz $\mathrm{S}$, et al. The Lgr5 intestinal stem cell signature: robust expression of proposed quiescent ' +4 ' cell markers. EMBO J. 2012;31:3079-91.

31. He TC, Sparks AB, Rago C, Hermeking H, Zawel L, da Costa LT, et al. Identification of c-MYC as a target of the APC pathway. Science. 1998;281:1509-12.

32. Ordonez-Moran P, Dafflon C, Imajo M, Nishida E, Huelsken J. HOXA5 counteracts stem cell traits by inhibiting Wnt signaling in colorectal cancer. Cancer Cell. 2015;28:815-29.

33. Moll R, Zimbelmann R, Goldschmidt MD, Keith M, Laufer J, Kasper M, et al. The human gene encoding cytokeratin 20 and its expression during fetal development and in gastrointestinal carcinomas. Differentiation. 1993;53:75-93.

34. Moschos SJ, Jukic DM, Athanassiou C, Bhargava R, Dacic S, Wang $\mathrm{X}$, et al. Expression analysis of Ubc9, the single small ubiquitin-like modifier (SUMO) E2 conjugating enzyme, in normal and malignant tissues. Hum Pathol. 2010;41:1286-98.

35. Tang Z, Li C, Kang B, Gao G, Li C, Zhang Z. GEPIA: a web server for cancer and normal gene expression profiling and interactive analyses. Nucleic Acids Res. 2017;45:W98-W102.

36. Decque A, Joffre O, Magalhaes JG, Cossec JC, Blecher-Gonen R, Lapaquette $\mathrm{P}$, et al. Sumoylation coordinates the repression of 
inflammatory and anti-viral gene-expression programs during innate sensing. Nat Immunol. 2016;17:140-9.

37. Mustfa SA, Singh M, Suhail A, Mohapatra G, Verma S, Chakravorty $\mathrm{D}$, et al. SUMOylation pathway alteration coupled with downregulation of SUMO E2 enzyme at mucosal epithelium modulates inflammation in inflammatory bowel disease. Open Biol. 2017;7 https://doi.org/10.1098/rsob.170024.

38. Suhail A, Rizvi ZA, Mujagond P, Ali SA, Gaur P, Singh M, et al. DeSUMOylase SENP7-mediated epithelial signaling triggers intestinal inflammation via expansion of gamma-delta T cells. Cell Rep. 2019;29:3522-38.e3527.

39. Bu P, Wang L, Chen KY, Srinivasan T, Murthy PK, Tung KL, et al. A miR-34a-numb feedforward loop triggered by inflammation regulates asymmetric stem cell division in intestine and colon cancer. Cell Stem Cell. 2016;18:189-202.

40. Lindemans CA, Calafiore M, Mertelsmann AM, O'Connor MH, Dudakov JA, Jenq RR, et al. Interleukin-22 promotes intestinalstem-cell-mediated epithelial regeneration. Nature. 2015;528:560-4.

41. Grivennikov SI, Greten FR, Karin M. Immunity, inflammation, and cancer. Cell. 2010;140:883-99.

42. Karin M, Clevers H. Reparative inflammation takes charge of tissue regeneration. Nature. 2016;529:307-15.

43. Cheloufi S, Elling U, Hopfgartner B, Jung YL, Murn J, Ninova M, et al. The histone chaperone CAF-1 safeguards somatic cell identity. Nature. 2015;528:218-24.

44. Cossec JC, Theurillat I, Chica C, Bua Aguin S, Gaume X, Andrieux A, et al. SUMO safeguards somatic and pluripotent cell identities by enforcing distinct chromatin states. Cell Stem Cell. 2018;23:742-57.e748.

45. Tian H, Biehs B, Warming S, Leong KG, Rangell L, Klein OD, et al. A reserve stem cell population in small intestine renders Lgr5-positive cells dispensable. Nature. 2011;478:255-9.

46. van Es JH, Sato T, van de Wetering M, Lyubimova A, Yee Nee AN, Gregorieff A, et al. Dll1 + secretory progenitor cells revert to stem cells upon crypt damage. Nat Cell Biol. 2012;14:1099-104.

47. Buczacki SJ, Zecchini HI, Nicholson AM, Russell R, Vermeulen $\mathrm{L}$, Kemp R, et al. Intestinal label-retaining cells are secretory precursors expressing Lgr5. Nature. 2013;495:65-9.

48. Basak O, van de Born M, Korving J, Beumer J, van der Elst S, van Es JH, et al. Mapping early fate determination in Lgr5+ crypt stem cells using a novel Ki67-RFP allele. EMBO J. 2014;33:2057-68.
49. Tetteh PW, Basak O, Farin HF, Wiebrands K, Kretzschmar K, Begthel H, et al. Replacement of lost Lgr5-positive stem cells through plasticity of their enterocyte-lineage daughters. Cell Stem Cell. 2016;18:203-13.

50. Schwitalla S, Fingerle AA, Cammareri P, Nebelsiek T, Goktuna SI, Ziegler PK, et al. Intestinal tumorigenesis initiated by dedifferentiation and acquisition of stem-cell-like properties. Cell. 2013;152:25-38.

51. Pierce AJ, Johnson RD, Thompson LH, Jasin M. XRCC3 promotes homology-directed repair of DNA damage in mammalian cells. Genes Dev. 1999;13:2633-8.

52. Sato T, Vries RG, Snippert HJ, van de Wetering M, Barker N, Stange DE, et al. Single Lgr5 stem cells build crypt-villus structures in vitro without a mesenchymal niche. Nature. 2009;459:262-5.

53. Carvalho BS, Irizarry RA. A framework for oligonucleotide microarray preprocessing. Bioinformatics. 2010;26:2363-7.

54. Ritchie ME, Phipson B, Wu D, Hu Y, Law CW, Shi W, et al. limma powers differential expression analyses for RNA-sequencing and microarray studies. Nucleic Acids Res. 2015;43:e47.

55. Ashburner M, Ball CA, Blake JA, Botstein D, Butler H, Cherry JM, et al. Gene ontology: tool for the unification of biology. The Gene Ontology Consortium. Nat Genet. 2000;25:25-9.

56. The Gene Ontology Consortium. The Gene Ontology resource: 20 years and still GOing strong. Nucleic Acids Res. 2019;47: D330-8.

57. Fabregat A, Jupe S, Matthews L, Sidiropoulos K, Gillespie M, Garapati P, et al. The reactome pathway knowledgebase. Nucleic Acids Res. 2018;46:D649-55.

58. Kanehisa M, Furumichi M, Tanabe M, Sato Y, Morishima K. KEGG: new perspectives on genomes, pathways, diseases and drugs. Nucleic Acids Res. 2017;45:D353-61.

59. Ogata H, Goto S, Sato K, Fujibuchi W, Bono H, Kanehisa M. KEGG: Kyoto Encyclopedia of Genes and Genomes. Nucleic Acids Res. 1999;27:29-34.

60. Subramanian A, Tamayo P, Mootha VK, Mukherjee S, Ebert BL, Gillette MA, et al. Gene set enrichment analysis: a knowledgebased approach for interpreting genome-wide expression profiles. Proc Natl Acad Sci USA. 2005;102:15545-50.

61. Chen J, Bardes EE, Aronow BJ, Jegga AG. ToppGene Suite for gene list enrichment analysis and candidate gene prioritization. Nucleic Acids Res. 2009;37:W305-311. 